\title{
Erratum
}

\section{Functional determinants and geometry}

\section{Robin Forman}

Department of Mathematics, Rice University, Houston, TX 77251, USA

Oblatum 11-VII-1991

The author would like to thank D. Burghelea and T. Kappeler for pointing out the existence of an error in the paper Functional determinants and geometry, Invent. Math. 88, 447-493 (1987). The error occurs in part 2 of Lemma 3.1, which should read:

2. If $n$ is odd then for each $x$ there is some projection $\pi_{x}$ such that

$$
\lim _{s \rightarrow 0}\left(L^{-s} f\right)(x)=\frac{1}{n}\left[\pi_{x} f(x+)+\left(1-\pi_{x}\right) f(x-)\right] .
$$

The rest of the lemma remains as is. The mistake occurs at the top of p. 478, which should read:

$$
\lim _{s \rightarrow 0} \pm \sum_{k=1}^{\infty} \frac{1}{i(k)^{1+s n}}\left(e^{i \frac{\pi}{2} s}-e^{-i \frac{\pi}{2} s}\right)=\lim _{s \rightarrow 0} \pm\left(2 \sin \left(\frac{\pi}{2} s\right)\right) \sum_{k=1}^{\infty} \frac{1}{k^{1+s n}}
$$

with the sign being +1 or -1 if $n \equiv 1(\bmod 4)$ or $n \equiv 3(\bmod 4)$ resp. The simple estimate

$$
\frac{1}{s n}=\int_{1}^{\infty} x^{-(1+s n)} d x<\sum_{k=1}^{\infty} \frac{1}{k^{1+s n}}<1+\int_{1}^{\infty} x^{-(1+s n)} d x=1+\frac{1}{s n}
$$

shows that

$$
\lim _{s \rightarrow 0}\left(L^{-s} f\right)(x)=\lim _{s \rightarrow 0} \pm\left(2 \sin \left(\frac{\pi}{2} s\right)\right) \sum_{k=1}^{\infty} \frac{1}{k^{1+s n}}= \pm \frac{\pi}{n}=\frac{1}{n} \lim _{x \rightarrow \pi \mp} h(x) .
$$

The remainder of the proof proceeds as before. There is a corresponding change in the definition of $R(x)$ given in formula (4)

$$
R(x)= \begin{cases}\frac{1}{2} & \text { if } n \text { is even } \\ \frac{\pi_{x}}{n} & \text { if } n \text { is odd }\end{cases}
$$


Similarly, in the case of $n$ odd, in all formulae between statement (4) (bottom p. 478) and statement (5) (top p. 480), the term

$$
(1-R(x))
$$

should be replaced by

$$
\left(\frac{1}{n}-R(x)\right)=\frac{1-\pi_{x}}{n} .
$$

These changes do not at all affect the examples considered in the paper. 